\title{
Budd-Chiari syndrome with antiphospholipid syndrome and systemic lupus erythematosus in a patient with Klinefelter's syndrome
}

\author{
Mingee Lee ${ }^{1}$, Jin-Young Huh", Ji-Hyang Lee', Sun-myoung Kang ${ }^{1}$, Jae-Yong Lee ${ }^{1}$, \\ Oh-Chan Kwon', Eun $\mathrm{Na} \mathrm{Kim}{ }^{2}$, Jihun Kim², Danbi Lee ${ }^{1}$ \\ Departments of ${ }^{1}$ Internal Medicine and ${ }^{2}$ Pathology, Asan Medical Center, University of Ulsan College of Medicine, Seoul, Korea
}

Klinefelter's syndrome is the most common congenital abnormality that causes primary hypogonadism. It is associated with diseases that predominantly affect women, such as systemic lupus erythematosus (SLE), and it can sometimes cause veno-occlusive disease. We experienced a case of Budd-Chiari syndrome (BCS) in a 33-year-old man with Klinefelter's syndrome presented with hematemesis and edema in both lower extremities. The clinical and laboratory findings were compatible with SLE, antiphospholipid syndrome, and BCS. To the best of our knowledge, this is the first case report to describe a simultaneous presentation of these four clinical syndromes in a single patient.

Keywords: Klinefelter's syndrome; Systemic lupus erythematosus; Antiphospholipid syndrome; Budd-Chiari syndrome; Liver cirrhosis

\section{INTRODUCTION}

Klinefelter's syndrome is the most common congenital hypogonadal disease, occurring in 1 in 1,000 live male births. The association between Klinefelter's syndrome and autoimmune disease - particularly systemic lupus erythematosus (SLE) - has been reported several times since it was first described by Oritz-Neu and LeRoy in 1969 [1]. SLE is a typical autoimmune disease characterized by multisystem involvement and is associated with antiphospholipid syndrome (APS) in about $36 \%$ of patients [2]. APS is characterized by recurrent events of arterial and venous thrombosis, fetal loss, and thrombocytopenia. Budd-Chiari syndrome (BCS) secondary to APS

Received: July 6, 2016, Revised: August 28, 2016 Accepted: August 30, 2016

Corresponding Author: Danbi Lee, Division of Gastroenterology, Department of Internal Medicine, Asan Medical Center, University of Ulsan College of Medicine, 88 Olympic-ro 43-gil, Songpa-gu, Seoul 05505, Korea

Tel: +82-2-3010-3907, Fax: +82-2-476-0824

E-mail: leighdb@hanmail.net has also been reported [3]. Herein, we report a rare case of a single 33-year-old male patient with Klinefelter's syndrome presenting with BCS secondary to APS.

\section{CASE}

A 33-year-old man presented at our emergency room with massive hematemesis. He had been diagnosed with Klinefelter's syndrome (Fig. 1) at the age of 14, for which he had received testosterone replacement therapy. Although he decided to discontinue this treatment 3 years prior to admission, he had resumed it 6 months before coming to our hospital. Three months before admission, he was diagnosed with SLE due to the occurrence of alopecia, oral ulcer, proteinuria, ANA, and anti-ds-DNA; he had started taking hydroxychloroquine. He claimed that he did not take any other medications, including herbal medications, and he did not consume alcohol on a regular basis.

He had hepatosplenomegaly and pretibial pitting edema. His laboratory results were as follows: white blood cell count,

Copyright ( 12017 Yeungnam University College of Medicine

This is an Open Access article distributed under the terms of the Creative Commons Attribution Non-Commercial License (http://creativecommons.org/licenses/by-nc/4.0/) which permits unrestricted non-commercial use, distribution, and reproduction in any medium, provided the original work is properly cited. 


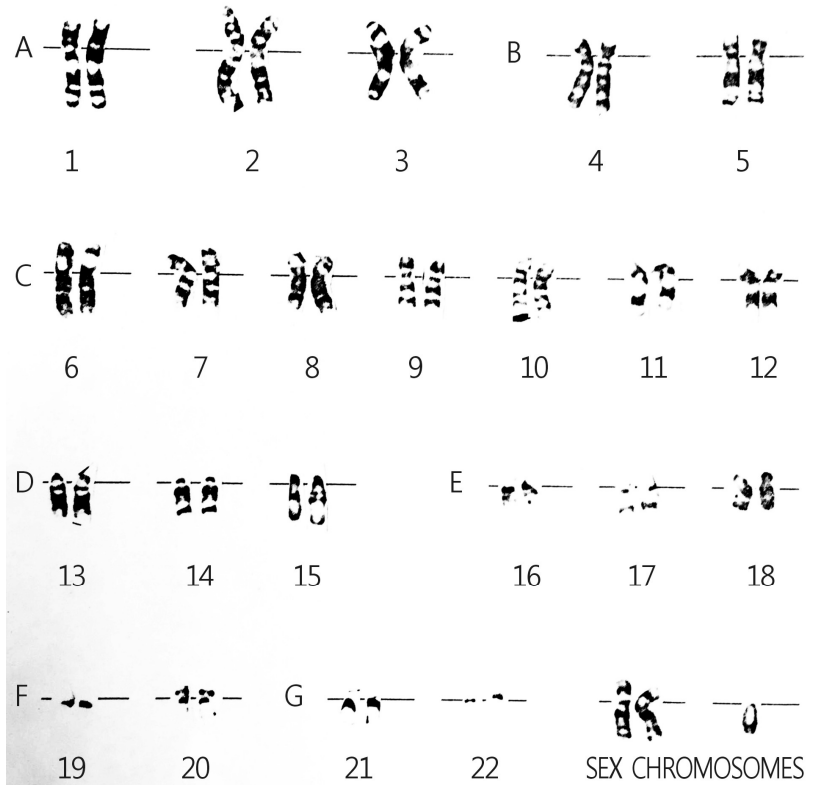

Fig. 1. Chromosome analysis of the patient with Klinefelter's syndrome. The karyotype in this case was 47, XXY.
6,900/ $\mathrm{mm}^{3}$ (neutrophil 63.9\%, lymphocyte 22.7\%); hemoglobin, $7.3 \mathrm{~g} / \mathrm{dL}$; and platelet count, $136,000 / \mathrm{mm}^{3}$. His aspartate aminotransferase level was $33 \mathrm{IU} / \mathrm{L}$, alanine aminotransferase was $14 \mathrm{IU} / \mathrm{L}$, alkaline phosphatase was $82 \mathrm{IU} / \mathrm{L}$, total bilirubin was $4.1 \mathrm{mg} / \mathrm{dL}$, direct bilirubin was $3.1 \mathrm{mg} / \mathrm{dL}$, serum creatinine was $0.69 \mathrm{mg} / \mathrm{dL}$, and albumin was $2.2 \mathrm{~g} / \mathrm{dL}$. The prothrombin time was $17.9 \mathrm{~s}$ (range, 10-13 s).

To clarify the cause of hematemesis, we performed esophagogastroduodenoscopy and abdominal computed tomography (CT). The esophageal endoscopy showed tortuous varices with blood clots and red color signs. We, therefore, treated esophageal varices with band ligation (Fig. 2). Abdominal CT (Fig. 3A, 3B) showed hepatomegaly with liver cirrhosis, splenomegaly, poor opacification of hepatic veins, and extensive portosystemic shunting.

We performed an additional diagnostic work-up to determine the cause of liver cirrhosis. According to the laboratory findings, viral hepatitis, such as hepatitis B or hepatitis C,
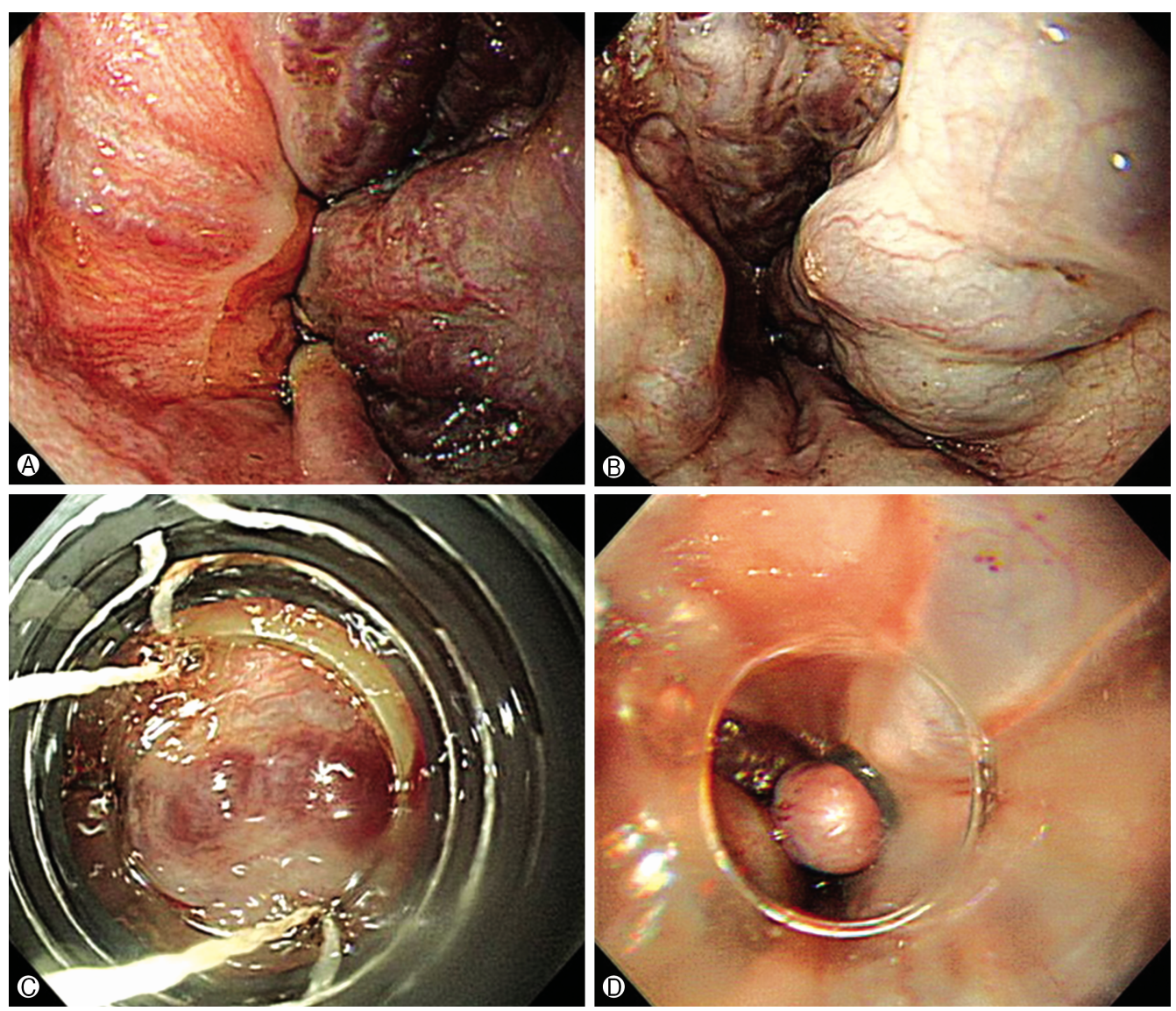

Fig. 2. Esophagogastroduodenoscopy. (A, B) Four strands of large beaded venous distensions extended up to the upper esophagus with red color signs. (C, D) Four rubber bands were tightly applied. 
was ruled out because serum hepatitis B surface (HBs) antigen and anti-HBs were negative and positive, respectively, and anti-hepatitis $\mathrm{C}$ virus was negative. The immunologic marker results were negative for anti-mitochondrial antibody, antismooth muscle antibody, and anti-liver kidney microsome antibody [1]. However, anti-nuclear antibody was positive (nucleolar type, 1:640). The anti-ds-DNA antibody level was 8.0 $\mathrm{IU} / \mathrm{mL}$ (range, $0-7 \mathrm{IU} / \mathrm{mL}$ ). The serum compliment 3 (C3) and C4 levels were $63.6 \mathrm{mg} / \mathrm{dL}$ (range, 90-180 mg/dL) and 11.3 $\mathrm{mg} / \mathrm{dL}$ (range, $10-40 \mathrm{mg} / \mathrm{dL}$ ), respectively. The level of anticardiolipin antibody immunoglobulin $\mathrm{G}(\mathrm{IgG})$ was $78 \mathrm{IgG}$ phospholipid units (GPL)/mL (range, $<20 \mathrm{GPL} / \mathrm{mL}$ ). The lupus anticoagulant and direct coombs test results were both positive. The anti-beta-2-glycoprotein I IgG and IgM readings were 114 (range, $<20 \mathrm{G}$ units) and 66 (range, $<20 \mathrm{M}$ units), respectively. Moreover, anti-SSA (Ro) was positive, but the other
anti-ENAs were all negative. These results confirmed the initial SLE diagnosis. In addition, we also considered the diagnosis of autoimmune hepatitis.

We performed a transjugular liver biopsy (TLB) due to the prothrombin time prolongation. We tried to obtain a liver histologic finding and to measure the hepatic venous pressure gradient to exclude the possibility of autoimmune hepatitis and non-cirrhotic portal hypertension. However, TLB failed because cavography revealed hepatic vein occlusion (Fig. 3C). Thus, we attempted a liver biopsy via the transhepatic approach. The subsequent histologic findings (Fig. 3D) revealed reversed lobulation cirrhosis, diffuse sinusoidal dilatation, and perivenular sclerosis, indicating venous outflow obstruction. These findings are consistent with BCS with secondary APS.

In conclusion, our patient originally had Klinefelter's syndrome and SLE. His secondary APS caused BCS, which led
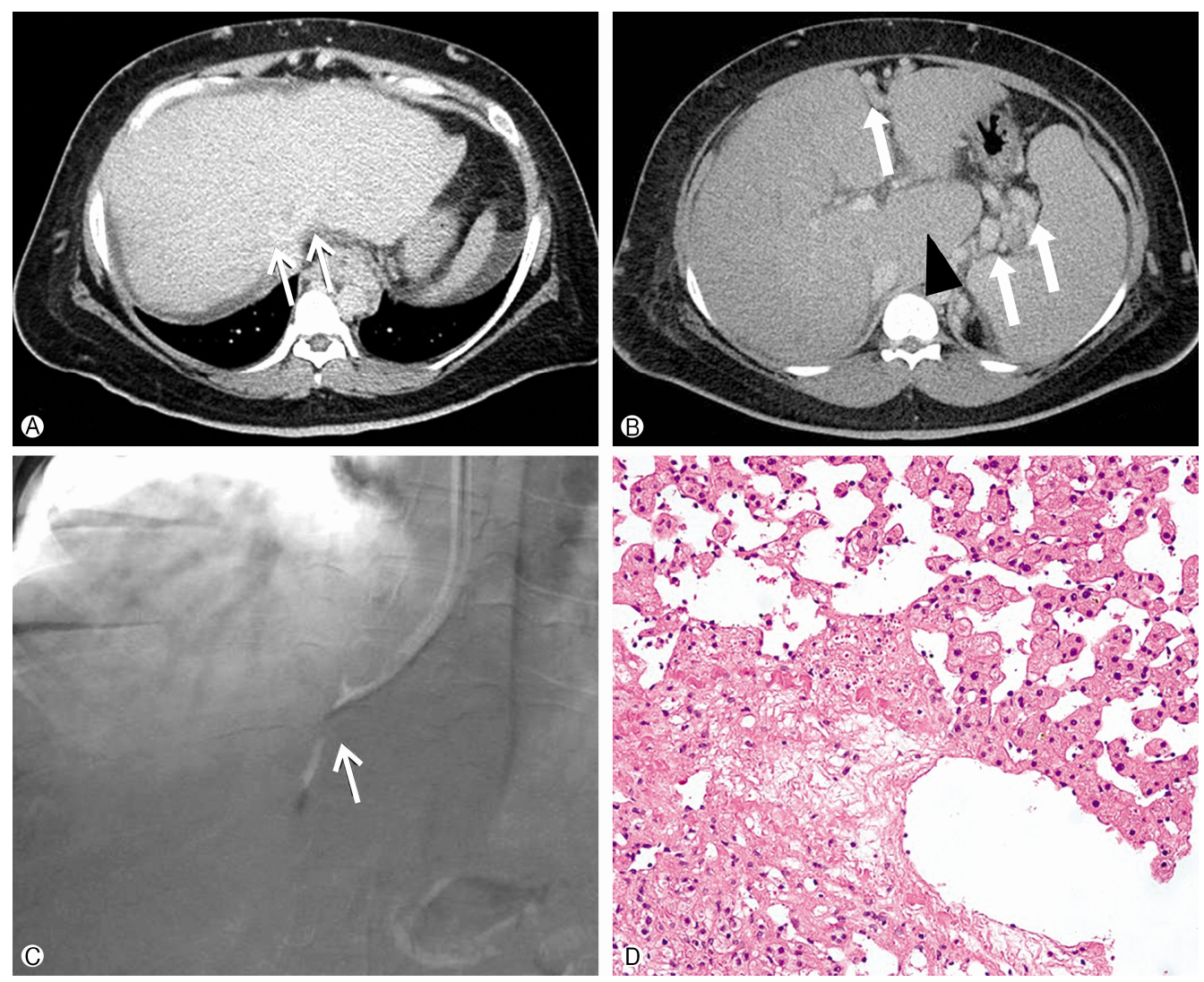

Fig. 3. Representative radiologic and pathologic images of the study patient. (A, B) Contrast-enhanced transverse computed tomography obtained during the delayed phase showed poor opacification of the hepatic vein (black arrow), enlarged caudate lobe (black arrow head), and collateral vessels (white arrow). (C) Cavography via the internal jugular vein. A transjugular liver biopsy was attempted by three radiologists, but could not be performed, possibly due to proximal hepatic vein stenosis or occlusion. (D) A liver needle biopsy shows a diffuse sinusoidal dilatation surrounding the central vein. 
to liver cirrhosis with esophageal varices. $\mathrm{He}$ is awaiting a liver transplantation due to a deteriorating hepatic function and recurrent esophageal variceal bleeding.

\section{DISCUSSION}

BCS is a rare, but potentially lethal, disease affecting 1 per million individuals. It is characterized by an obstruction of the hepatic venous outflow. The syndrome usually presents with abdominal pain, hepatomegaly, and ascites. Various causes have been identified, and commonly acquired causes are myeloproliferative disorders, paroxysmal nocturnal hemoglobinuria, APS, cancer, and pregnancy [4].

APS is an autoimmune disease characterized by arterial and venous thromboembolic events and pregnancy morbidity. Although antiphospholipid antibodies are rarely found in patients with Klinefelter's syndrome, they can be found in association with SLE. The association between Klinefelter's syndrome and SLE has been well established. In 2008, Scofield et al. [5] reported an incidence of SLE in patients with Klinefelter's syndrome as approximately 1 in 960. Another report reviewed 43 patients with BCS secondary to APS [6], and two-thirds of patients were female and had BCS as a first clinical manifestation of APS.

An increased rate of veno-occlusive disease (VOD) has also been reported in patients with Klinefelter's syndrome. Campbell and Price [7] found that the risk of deep venous thrombosis or pulmonary embolism was 5-20 times higher in patients with Klinefelter's syndrome.

The high incidence of autoimmune disorder and VOD in Klinefelter's syndrome is attributed to an altered hormone level and is explained by an X chromosome gene dose effect. Some studies showed that the androgen replacement treatment for Klinefelter's syndrome can suppress immunological parameters, reduce the severity of SLE, and resolve recurrent hypostatic lower extremity ulcerations [8-10]. Other studies have revealed an association between low testosterone levels and high incidence of autoimmune disease [11,12]. In the case of our patient, he had been taking testosterone undecanoate since he was 14 years old with no significant side effects. However, he arbitrarily stopped taking this medication and was lost to follow up for 2 years, which may have contributed to the development of autoimmune disease.

In summary, we report the first case of BCS with secondary
APS and SLE in a Kline-felter's syndrome patient. Autoimmune disorder and VOD are more prevalent, and careful evaluation is necessary in these patients. Additionally, androgen supplement may be considered as a possible preventive and therapeutic option for autoimmune disease.

\section{CONFLICT OF INTEREST}

No potential conflict of interest relevant to this article was reported.

\section{ORCID}

Mingee Lee, https://orcid.org/0000-0002-0106-2428

Danbi Lee, https://orcid.org/0000-0002-8336-0006

\section{REFERENCES}

1. Ortiz-Neu C, LeRoy EC. The coincidence of Klinefelter's syndrome and systemic lupus erythematosus. Arthritis Rheum 1969;12:241-6.

2. Love PE, Santoro SA. Antiphospholipid antibodies: anticardiolipin and the lupus anticoagulant in systemic lupus erythematosus (SLE) and in non-SLE disorders. Prevalence and clinical significance. Ann Intern Med 1990;112:682-98.

3. Pelletier S, Landi B, Piette JC, Ekert P, Coutellier A, Desmoulins $\mathrm{C}$, et al. Antiphospholipid syndrome as the second cause of non-tumorous Budd-Chiari syndrome. J Hepatol 1994;21:76-80.

4. Menon KV, Shah V, Kamath PS. The Budd-Chiari syndrome. N Engl J Med 2004;350:578-85.

5. Scofield RH, Bruner GR, Namjou B, Kimberly RP, RamseyGoldman R, Petri M, et al. Klinefelter's syndrome (47, XXY) in male systemic lupus erythematosus patients: support for the notion of a gene-dose effect from the $\mathrm{X}$ chromosome. Arthritis Rheum 2008;58:2511-7.

6. Espinosa G, Font J, García-Pagan JC, Tàssies D, Reverter JC, Gaig C, et al. Budd-Chiari syndrome secondary to antiphospholipid syndrome: clinical and immunologic characteristics of 43 patients. Medicine (Baltimore) 2001;80:345-54.

7. Campbell WA, Price WH. Venous thromboembolic disease in Klinefelter's syndrome. Clin Genet 1981;19:275-80.

8. Koçar IH, Yesilova Z, Ozata M, Turan M, Sengül A, Ozdemir I. The effect of testosterone replacement treatment on immunological features of patients with Klinefelter's syndrome. Clin Exp Immunol 2000;121:448-52.

9. Igawa K, Nishioka K. Leg ulcer in Klinefelter's syndrome. J Eur Acad Dermatol Venereol 2003;17:62-4.

10. Bizzarro A, Valentini G, Di Martino G, DaPonte A, De Bellis A, Iacono G. Influence of testosterone therapy on clinical and 
Mingee Lee et al.

immunological features of autoimmune diseases associated with Klinefelter's syndrome. J Clin Endocrinol Metab 1987; 64:32-6.

11. Spector TD, Ollier W, Perry LA, Silman AJ, Thompson PW, Edwards A. Free and serum testosterone levels in 276 males: a comparative study of rheumatoid arthritis, ankylosing spon- dylitis and healthy controls. Clin Rheumatol 1989;8:37-41.

12. Jiménez-Balderas FJ, Tápia-Serrano R, Fonseca ME, Arellano J, Beltrán A, Yáñez P, et al. High frequency of association of rheumatic/autoimmune diseases and untreated male hypogonadism with severe testicular dysfunction. Arthritis Res 2001;3:362-7. 